\title{
Robust Delay Independent Stability Analysis for the Switched Interval Time-Delay Systems with Time-Driven Switching Strategy
}

\author{
Juing-Shian Chiou ${ }^{1}$ and Jen-Hsing $\mathrm{Li}^{2}$ \\ ${ }^{1}$ Department of Electrical Engineering, Southern Taiwan University of Science and Technology, Tainan, Taiwan \\ ${ }^{2}$ Department of Electrical Engineering, Kun Shan University, Tainan, Taiwan \\ Correspondence should be addressed to Juing-Shian Chiou; jschiou@mail.stust.edu.tw
}

Received 13 July 2013; Accepted 23 September 2013

Academic Editor: Jui-Sheng Lin

Copyright ( $\odot 2013$ J.-S. Chiou and J.-H. Li. This is an open access article distributed under the Creative Commons Attribution License, which permits unrestricted use, distribution, and reproduction in any medium, provided the original work is properly cited.

Some new criteria of delay independent stability for the switched interval time-delay systems are deduced. The switching structure does depend on time-driven switching strategies. The total activation time ratio of the switching law can be determined to guarantee that the switched interval time-delay system is exponentially stable.

\section{Introduction}

Switched systems constitute an important class of hybrid systems. Such systems can be described by a family of continuous-time subsystems (or discrete-time subsystems) and a rule that orchestrates the switching between them. It is well known that a wide class of physical systems in power systems, chemical process control systems, navigation systems, automobile speed change system, and so forth may be appropriately described by the switched model [1-7]. In the study of switched systems, most works have been centralized on the problem of stability. In the last two decades, there has been increasing interest in the stability analysis for such switched systems; see, for example, [8, 9] and the references cited therein. Two important methods are used to construct the switching law for the stability analysis of the switched systems. One is the state-driven switching strategy [9]; the other is the time-driven switching strategy [8].

The state-driven switching method is that if all subsystems have the common Lyapunov function or the multiple Lyapunov functions, there are many choices of switching strategy to make the whole system stable. However, using these kinds of methods, the system must meet conditions completely. Therefore, the common Lyapunov function or the multi-Lyapunov function is difficult to construct for practical systems; even if we can construct the function, it is more complicated and not easy to implement on practical systems.

The time-driven switching method is based on the concept of dwell time [2] that when all subsystem matrices are Hurwitz stable, then the entire switched system is exponentially stable for any switching signal if the time between consecutive switching (dwell time) is sufficiently large. [10] that switching among stable linear systems results in a stable system provided that switching is slow-on-the-average. But in many applications, unstable subsystems of switched systems cannot be avoided in fact [11]. If the average dwell time is chosen sufficiently large, and the total activation time of unstable subsystems is relatively small compared with that of Hurwitz stable subsystems, then exponential stability of a desire degree is guaranteed.

Furthermore, the time-delay phenomenon also cannot be avoided in practical systems, for instance, chemical process, long distance transmission line, hybrid procedure, electron network, and so forth. The problem of time-delay may cause instability and poor performance of practical systems [1214]. Therefore, the stability analysis of switched systems with time delay is very worthy to be researched. In a control system, uncertainties may be due to measure errors, modeling errors, linearization approximations, and so forth. There seem to be some alternatives in formulating uncertainties or 
perturbations. $[13,15]$ shared the formulation which systems matrices are assumed to be perturbation. However, it does not take the position that the system matrices are expressed in as the sum of the two terms, but that the bounds for them are somehow known. These systems are called interval timedelay systems. The goal of this paper is to derive some robust sufficient stability conditions for the switched interval timedelay system.

Basically, current efforts to achieve stability in time-delay systems can be divided into two categories, namely, delayindependent criteria and delay-dependent criteria. In this paper, in view of delay-independent analysis, we expect to aid in studying stability and designing time-driven switching law to achieve and implement in a practical switched interval time-delay system.

The following notations will be used throughout the paper: $\lambda(A)$ stands for the eigenvalues of matrix $A$, $\|A\|$ denotes the norm of matrix $A$; that is, $\|A\|=$ $\operatorname{Max}\left[\lambda\left(A^{T} A\right)\right]^{1 / 2}$, and $\mu(A)$ means the matrix measure of matrix $A$; that is, $\mu(A)=\operatorname{Max}\left[\lambda\left(\left(A+A^{T}\right) / 2\right)\right]$. $\|A\|=$ $\operatorname{Max}\left[\lambda\left(A^{T} A\right)\right]^{1 / 2}$.

\section{System Description and Problem Statement}

First, consider the following switched time-delay system

$$
\begin{gathered}
\dot{x}(t)=A_{\sigma(t)} x(t)+B_{\sigma(t)} x(t-\tau), \\
x\left(t_{0}\right)=x_{0}, \quad x(t)=\psi(t), \quad t \in[-\tau, 0],
\end{gathered}
$$

where $x(t) \in \mathbf{R}^{n}$ is state, $A_{\sigma(t)} \in \mathbf{R}^{n \times n}, B_{\sigma(t)} \in \mathbf{R}^{n \times n}$, $t_{0} \geq 0$ is the initial time, $x_{0}$ is the initial state, and $\sigma(t)$ : $\left[t_{0}, \infty\right) \rightarrow\{1,2, \ldots, N\}$ is a piecewise constant function of time, called a switch signal; that is, the matrix $A_{\sigma(t)}$ switches between matrices $A_{1}, A_{2}, \ldots, A_{N}$ belonging to the set $\mathbf{A} \equiv$ $\left\{A_{1}, A_{2}, \ldots, A_{N}\right\}$ and $A_{i}, i \in\{1,2, \ldots, N\}$; the matrix $B_{\sigma(t)}$ switches between matrices $B_{1}, B_{2}, \ldots, B_{N}$ belonging to the set $B \equiv\left\{B_{1}, B_{2}, \ldots, B_{N}\right\}$ and $B_{i}, i \in\{1,2, \ldots, N\} . \tau>0$ is the time-delay duration. $\psi(t)$ is a vector-valued initial continuous function defined on the interval $[-\tau, 0]$, and finally $\psi(t)$, defined on $-\tau \leq t \leq 0$, is the initial condition of the state.

Let us consider the switched interval time-delay system described by

$$
\dot{x}(t)=A_{i}^{I} x(t)+B_{i}^{I} x(t-\tau), \quad i \in\{1,2, \ldots, N\},
$$

where $A_{i}^{I}$ and $B_{i}^{I}$ are matrices whose elements vary in prescribed defined as

$$
A_{i}^{I}=\left[a_{k l i}\right], \quad B_{i}^{I}=\left[b_{k l i}\right]
$$

where $k, l=1,2, \ldots, n . \underline{a}_{k l_{-i}} \leq a_{k l_{i}} \leq \bar{a}_{k l_{i}}$ and $\underline{b}_{k l i} \leq b_{k l_{i}} \leq$ $\bar{b}_{k l i}$.

Denote:

$$
\begin{array}{ll}
\underline{A}_{i}=\left[\underline{a}_{k l i}\right], & \bar{A}_{i}=\left[\bar{a}_{k l i}\right], \\
\underline{B}_{i}=\left[\underline{b}_{k l i}\right], & \bar{B}_{i}=\left[\bar{b}_{k l i}\right],
\end{array}
$$

and let

$$
A_{i}=\frac{\underline{A}_{i}+\bar{A}_{i}}{2}, \quad B_{i}=\frac{\underline{B}_{i}+\bar{B}_{i}}{2},
$$

where $A_{i}$ and $B_{i}$ are the average matrices between $\underline{A}_{i}, \bar{A}_{i}$, and $\underline{B}_{i}, \bar{B}_{i}$, respectively. Furthermore,

$$
A_{i}^{b}=A_{i}^{I}-A_{i}, \quad B_{i}^{b}=B_{i}^{I}-B_{i},
$$

where $A_{i}^{b}$ and $B_{i}^{b}$ are the bias matrices between $\bar{A}_{i}, A_{i}$, and $\bar{B}_{i}$, $B_{i}$, respectively. Also,

$$
A_{i}^{m}=\bar{A}_{i}-A_{i}, \quad B_{i}^{m}=\bar{B}_{i}-B_{i},
$$

where $A_{i}^{m}$ and $B_{i}^{m}$ are the maximal bias matrices between $\bar{A}_{i}$, $A_{i}$, and $\bar{B}_{i}, B_{i}$, respectively.

From the properties of matrix norm, we have

$$
\left\|A_{i}^{b}\right\| \leq\left\|A_{i}^{m}\right\|=\alpha_{i}, \quad\left\|B_{i}^{b}\right\| \leq\left\|B_{i}^{m}\right\|=\beta_{i},
$$

and denote

$$
\alpha_{I}=\max \left(\alpha_{i}\right), \quad \beta_{I}=\max \left(\beta_{i}\right),
$$

where $1 \leq i \leq N$.

In this paper, we study the robust stability analysis and switching law design for the switched interval time-delay systems.

\section{Delay-independent Stability Analysis}

Some helpful lemmas and definitions are given below.

Lemma 1 (see [16]). Consider the time-delay system:

$$
\dot{x}(t)=A x(t)+B x(t-\tau),
$$

where $x \in R^{n}, A$ and $B$ are matrices in proper dimensions, and $\tau$ is the delay duration. The stability of the time-delay system implies the stability for the following systems:

$$
\dot{w}(t)=(A+z B) w(t), \quad \forall|z|=1,
$$

and vice versa.

In the light of Lemma 1, for the switched time-delay system (1), all individual subsystems can be implied

$$
\dot{w}(t)=\left(A_{i}+z B_{i}\right) w(t)=\bar{A}_{i}(z) w(t), \quad \forall|z|=1 .
$$

Therefore, the system (12) is exponentially stable if and only if the switched time-delay system (1) is exponentially stable.

Lemma 2 (see [17]). For matrices $A \in \mathbf{R}^{n \times n}$ and $B \in \mathbf{R}^{n \times n}$, the following relation holds:

$$
\begin{aligned}
\|\exp [(A+z B) t]\| & \leq \exp [\mu(A+z B) t] \\
& \leq \exp [(\mu(A)+\|B\|) t], \quad \forall|z|=1 .
\end{aligned}
$$


Without loss of generality, we assume that the switched interval time-delay system (2) at least has one individual subsystem whose $\mu\left(A_{i}\right)+\left\|B_{i}\right\|+\alpha_{i}+\beta_{i}$ values are less than zero, the that of remaining individual system are not less than zero; that is,

$$
\begin{array}{r}
\mu\left(A_{i}\right)+\left\|B_{i}\right\|+\alpha_{i}+\beta_{i}<0, \quad 1 \leq i \leq r, \\
\mu\left(A_{i}\right)+\left\|B_{i}\right\|+\alpha_{i}+\beta_{i} \geq 0, \quad r+1 \leq i \leq N .
\end{array}
$$

Definition 3. Consider

$$
\begin{array}{ll}
\lambda_{\mathrm{doi}}^{-}=\min \left(\left|\mu\left(A_{i}\right)+\left\|B_{i}\right\|+\alpha_{i}+\beta_{i}\right|\right), & 1 \leq i \leq r, \quad(1 \\
\lambda_{\mathrm{doi}}^{+}=\max \left(\mu\left(A_{i}\right)+\left\|B_{i}\right\|+\alpha_{i}+\beta_{i}\right), & r+1 \leq i \leq N .
\end{array}
$$

Furthermore, we assume that $T^{+}(t)$ (or $\left.T^{-}(t)\right)$ is the total activation time of individual subsystems whose $\mu\left(A_{i}\right)+\left\|B_{i}\right\|+$ $\alpha_{i}+\beta_{i}$ values are not less than zero (total activation time of individual subsystems whose $\mu\left(A_{i}\right)+\left\|B_{i}\right\|+\alpha_{i}+\beta_{i}$ values are less than zero). The total activation time ratio between $T^{-}(t)$ and $T^{+}(t)$ can be called a switching law of the switched interval time-delay system (2). Therefore, we will find the ratio for the total activation time such that the switched interval time-delay system (2) is globally and exponentially stable with stability margin $\lambda$.

Theorem 4. Suppose that the switched interval time-delay system (2) exists in at least one individual subsystem whose $\mu\left(A_{i}\right)+\left\|B_{i}\right\|+\alpha_{i}+\beta_{i}$ value is less than zero. The switched interval time-delay system (2) is globally and exponentially stable with stability margin $\lambda$, if the system (2) satisfies the following switching law:

$$
\inf _{t \geq t_{0}}\left[\frac{T^{-}(t)}{T^{+}(t)}\right] \geq \frac{\left(\lambda_{d o i}^{+}+\lambda^{*}\right)}{\left(\lambda_{d o i}^{-}-\lambda^{*}\right)},
$$

where $\lambda \in\left(0, \lambda_{d o i}^{-}\right)$and $\lambda^{*} \in\left(\lambda, \lambda_{d o i}^{-}\right)$.

Proof. By Lemma 1, the stability of the switched interval time-delay system (2) can be transformed into the following system:

$$
\dot{w}(t)=\left(A_{i}^{I}+z B_{i}^{I}\right) w(t)=\bar{A}_{i}^{I} w(t) .
$$

The trajectory response of system (17) is written as follows:

$$
w(t)=e^{\bar{A}_{P_{i+1}}^{I}\left(t-t_{i}\right)} e^{\bar{A}_{p_{i}}^{I}\left(t_{i}-t_{i-1}\right)} \cdots e^{\bar{A}_{P_{1}}^{I}\left(t_{1}-t_{0}\right)} w\left(t_{0}\right) .
$$

In view of Lemma 2 , we can obtain the inequality

$$
\begin{aligned}
\|w(t)\| \leq & e^{\left(A_{P_{i+1}}^{I}+z B_{p_{i+1}}^{I}\right)\left(t-t_{i}\right)} \cdot e^{\left(A_{p_{i}}^{I}+z B_{p_{i}}^{I}\right)\left(t_{i}-t_{i-1}\right)} \\
& \cdots e^{A_{p_{1}}^{I}+z B_{p_{1}}^{I}\left(t_{1}-t_{0}\right)}\left\|w\left(t_{0}\right)\right\| \\
\leq & e^{\left[\mu\left(A_{P_{i+1}}^{I}\right)+\left\|B_{P_{i+1}}^{I}\right\|\right]\left(t-t_{i}\right)} \cdot e^{\left[\mu\left(A_{p_{i}}^{I}\right)+\left\|B_{P_{i}}^{I}\right\|\right]\left(t_{i}-t_{i-1}\right)} \\
& \cdots e^{\left[\mu\left(A_{p_{1}}^{I}\right)+\left\|B_{p_{1}}^{I}\right\|\right]\left(t_{1}-t_{0}\right)}\left\|w\left(t_{0}\right)\right\| .
\end{aligned}
$$

From the properties of matrix measure, we have

$$
\begin{gathered}
\mu\left(A_{i}^{I}\right) \leq \mu\left(A_{i}^{b}+A_{i}\right) \\
\leq \mu\left(A_{i}^{b}\right)+\mu\left(A_{i}\right) \leq \mu\left(A_{i}\right)+\alpha_{i}, \\
\left\|B_{i}^{I}\right\| \leq\left\|B_{i}\right\|+\beta_{i} .
\end{gathered}
$$

Hence, the inequality (19) can be written as

$$
\begin{aligned}
\|w(t)\| \leq & e^{\left[\mu\left(A_{p_{i+1}}\right)+\alpha_{p_{i+1}}+\left\|B_{p_{i+1}}\right\|+\beta_{p_{i+1}}\right]\left(t-t_{i}\right)} \\
& \cdots e^{\left[\mu\left(A_{p_{i}}\right)+\alpha_{p_{i}}+\left\|B_{p_{i}}\right\|+\beta_{p_{i}}\right]\left(t_{i}-t_{i-1}\right)} \\
& \cdots e^{\left[\mu\left(A_{p_{1}}\right)+\alpha_{p_{1}}+\left\|B_{p_{1}}\right\|+\beta_{p_{1}}\right]\left(t_{1}-t_{0}\right)}\left\|w\left(t_{0}\right)\right\| \\
\leq & e^{\lambda_{\mathrm{doi}}^{+} T^{+}-\lambda_{\mathrm{doi}}^{-} T^{-}}\left\|w\left(t_{0}\right)\right\| .
\end{aligned}
$$

Furthermore, the switching law (16) means that

$$
\begin{aligned}
\lambda_{\mathrm{doi}}^{+} T^{+}(t)-\lambda_{\mathrm{doi}}^{-} T^{-}(t) & \leq-\lambda^{*}\left(T^{+}(t)+T^{-}(t)\right) \\
& =-\lambda^{*}\left(t-t_{0}\right) .
\end{aligned}
$$

Finally, if we choose $\lambda \in\left(0, \lambda_{\mathrm{doi}}^{-}\right)$and $\lambda^{*} \in\left(0, \lambda_{\mathrm{doi}}^{-}\right)$, the following inequality can be obtained:

$$
\begin{aligned}
\|w(t)\| & \leq e^{-\lambda^{*}\left(t-t_{0}\right)}\left\|w\left(t_{0}\right)\right\| \\
& \leq e^{-\lambda\left(t-t_{0}\right)}\left\|w\left(t_{0}\right)\right\| .
\end{aligned}
$$

From the previous inequality (23), the system (17) is globally and exponentially stable with stability margin $\lambda$ and implies that the system (2) is also stable as the systems (17) and (2) have same stability as properties. Hence, the switched interval time-delay system (2) is also globally and exponentially stable with stability margin $\lambda$.

Remark 5. By Theorem 4, the stability condition of the switched interval time-delay system (1) is independent of time-delay.

\section{Example}

Example 1. Consider the switched interval time-delay system with interval matrices.

Subsystem 1. Consider

$$
\begin{aligned}
& A_{1}^{I}=\left[\begin{array}{cc}
{[-0.3,0.3} & {\left[\begin{array}{cc}
0.8, & 1.2
\end{array}\right]} \\
{[0.8,1.2} & {[-0.3,0.3}
\end{array}\right], \\
& B_{1}^{I}=\left[\begin{array}{ll}
{[-0.1,0.3} & {[-0.1,0.1} \\
{\left[\begin{array}{ll}
-0.1, & 0.1
\end{array}\right]} & {[-0.1,0.3}
\end{array}\right] .
\end{aligned}
$$

Subsystem 2. Consider

$$
\begin{gathered}
A_{2}^{I}=\left[\begin{array}{cc}
{[-3.1,} & -2.9 \\
{\left[\begin{array}{ll}
0.9, & 1.1
\end{array}\right]} & {\left[\begin{array}{ll}
-0.2, & 0.2
\end{array}\right]} \\
-3.3, & -2.7
\end{array}\right], \\
B_{2}^{I}=\left[\begin{array}{ll}
{\left[\begin{array}{ll}
-0.2, & 0
\end{array}\right]\left[\begin{array}{ll}
-0.1, & 0.1
\end{array}\right]} \\
{\left[\begin{array}{ll}
-0.4, & 0
\end{array}\right]} & {[-0.4,-0.2]}
\end{array}\right] .
\end{gathered}
$$




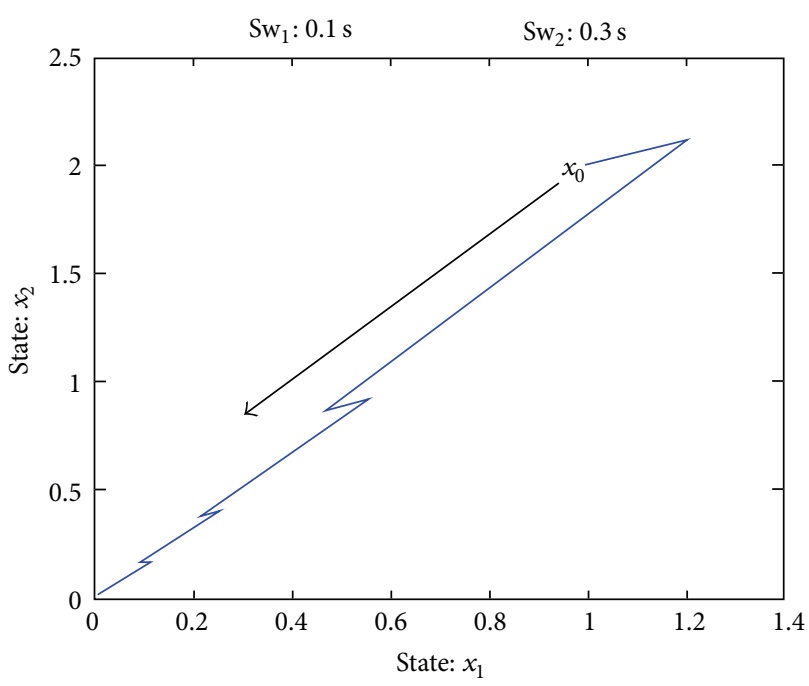

FIgURE 1: Trajectory response in Example.

From (5) and (7), we obtain the average matrices and maximal bias matrices.

Subsystem 1. Consider

$$
\begin{array}{cc}
A_{1}=\left[\begin{array}{ll}
0 & 1 \\
1 & 0
\end{array}\right], & B_{1}=\left[\begin{array}{cc}
0.1 & 0 \\
0 & 0.1
\end{array}\right], \\
A_{1}^{m}=\left[\begin{array}{ll}
0.3 & 0.2 \\
0.2 & 0.3
\end{array}\right], & B_{1}^{m}=\left[\begin{array}{ll}
0.2 & 0.1 \\
0.1 & 0.2
\end{array}\right] .
\end{array}
$$

Subsystem 2. Consider

$$
\begin{array}{ll}
A_{2}=\left[\begin{array}{cc}
-3 & 0 \\
1 & -3
\end{array}\right], & B_{2}=\left[\begin{array}{cc}
-0.1 & 0 \\
-0.2 & -0.3
\end{array}\right], \\
A_{2}^{m}=\left[\begin{array}{ll}
0.1 & 0.2 \\
0.1 & 0.3
\end{array}\right], & B_{2}^{m}=\left[\begin{array}{ll}
0.1 & 0.1 \\
0.2 & 0.1
\end{array}\right] .
\end{array}
$$

From (15a) and (15b), we can calculate $\lambda_{\text {doi }}^{+}=1.9$ and $\lambda_{\mathrm{doi}}^{-}=1.4867$. Finally, the total activation time ratio for the switching law is (with $\lambda=0.3, \lambda^{*}=0.6$ )

$$
\frac{T^{-}(t)}{T^{+}(t)} \geq \frac{\left(\lambda_{\mathrm{doi}}^{+}+\lambda^{*}\right)}{\left(\lambda_{\mathrm{doi}}^{-}-\lambda^{*}\right)}=2.8194
$$

In order to satisfy the switching law (27), we choose the total activation time ratio $3: 1$. The activation time of subsystem 1 is $0.1 \mathrm{sec}$, and the activation time of subsystem 2 is $0.3 \mathrm{sec}$, respectively. The trajectory of the switched interval time-delay system (for the average matrices $A_{1}, B_{1}, A_{2}$, and $B_{2}$ ) is shown in Figure 1 with initial state $\left[\begin{array}{ll}1 & 2\end{array}\right]^{T}$ and timedelay $0.1 \mathrm{sec}$.

\section{Conclusion}

We have developed methodologies for the delay-independent stability criteria of switched interval time-delay systems with time-driven switching strategy. On delay-independent stability analysis, the sufficient conditions of the switched laws are presented, and the total activation time ratio under the switching laws is required to be not less than a specified constant, such that the switched interval time-delay system is delay-independent and exponentially stable with stability margin. In addition, the main advantages of our approach showed that we can quantify the region of stability, extend to arbitrary subsystems of switched time-delay systems, and develop the simple time-driven switching rule to stabilize the switched interval time-delay systems.

\section{Acknowledgment}

This work is supported by the National Science Council, Taiwan, under Grants no. NSC 102-2221-E-218-017 and NSC1002632-E-218-001-MY3.

\section{References}

[1] D. Liberzon and A. S. Morse, "Basic problems in stability and design of switched systems," IEEE Control Systems Magazine, vol. 19, no. 5, pp. 59-70, 1999.

[2] J. S. Chiou and C. M. Cheng, "Stabilization analysis of the switched discrete-time systems using Lyapunov stability theorem and genetic algorithm," Chaos, Solitons and Fractals, vol. 42, no. 2, pp. 751-759, 2009.

[3] C. J. Wang and J. S. Chiou, "A stability condition with delaydependence for a class of switched large-scale time-delay systems," Journal of Applied Mathematics, vol. 2013, Article ID 360170, 7 pages, 2013.

[4] C. J. Wang and J. S. Chiou, "Stabilization analysis for the switched large-scale discrete-time systems via the state-driven switching," Discrete Dynamics in Nature and Society, vol. 2013, Article ID 630545, 5 pages, 2013.

[5] H. Sun and L. Hou, "Composite disturbance observer-based control and $H_{\infty}$ output tracking control for discrete-time switched systems with time-varying delay," Mathematical Problems in Engineering, vol. 2013, Article ID 698935, 12 pages, 2013.

[6] J. Wei, P. Shi, H. R. Karimi, and B. Wang, "BIBO stability analysis for delay switched systems with nonlinear perturbation," Abstract and Applied Analysis, vol. 2013, Article ID 738653, 8 pages, 2013.

[7] Z. He, X. Wang, Z. Gao, and J. Bai, "Sliding mode control based on observer for a class of state-delayed switched systems with uncertain perturbation," Mathematical Problems in Engineering, vol. 2013, Article ID 614878, 9 pages, 2013.

[8] J. S. Chiou, "Stability analysis for a class of switched largescale time-delay systems via time-switched method," IEE Proceedings-Control Theory \& Applications, vol. 153, no. 6, pp. 684-688, 2006.

[9] J. S. Chiou, C. J. Wang, C. M. Cheng, and C. C. Wang, "Analysis and synthesis of switched nonlinear systems using the T-S fuzzy model," Applied Mathematical Modelling, vol. 34, no. 6, pp. 14671481, 2010.

[10] J. P. Hespanha and A. S. Morse, "Stability of switched systems with average dwell-time," in Proceedings of the 38th IEEE Conference on Decision and Control (CDC '99), pp. 2655-2660, Phoenix, Ariz, USA, December 1999.

[11] G. Zhai, B. Hu, K. Yasuda, and A. N. Michel, "Stability analysis of switched systems with stable and unstable subsystems: an 
average dwell time approach," International Journal of Systems Science, vol. 32, no. 8, pp. 1055-1061, 2001.

[12] F. H. Hsiao, J. D. Hwang, and S. P. Pan, "D-stability analysis for discrete uncertain time-delay systems," Applied Mathematics Letters, vol. 11, no. 2, pp. 109-114, 1998.

[13] J. H. Park, "Simple criterion for asymptotic stability of interval neutral delay-differential systems," Applied Mathematics Letters, vol. 16, no. 7, pp. 1063-1068, 2003.

[14] G. Zhai, Y. Sun, X. Chen, and A. N. Michel, "Stability and $\mathrm{L}_{2}$ gain analysis for switched symmetric systems with time delay," in Proceedings of the American Control Conference, pp. 26822687, Denver, Colo, USA, June 2003.

[15] E. Tissir and A. Hmamed, "Stability tests of interval time delay systems," Systems \& Control Letters, vol. 23, no. 4, pp. 263-270, 1994.

[16] A. Hmamed, "Further results on the robust stability of uncertain time-delay systems," International Journal of Systems Science, vol. 22, no. 3, pp. 605-614, 1991.

[17] P. Lancaster, Theory of Matrices, Academic Press, New York, NY, USA, 1969. 


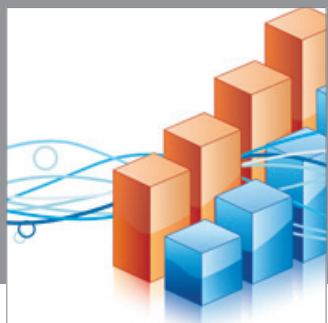

Advances in

Operations Research

mansans

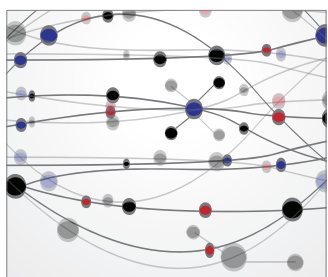

The Scientific World Journal
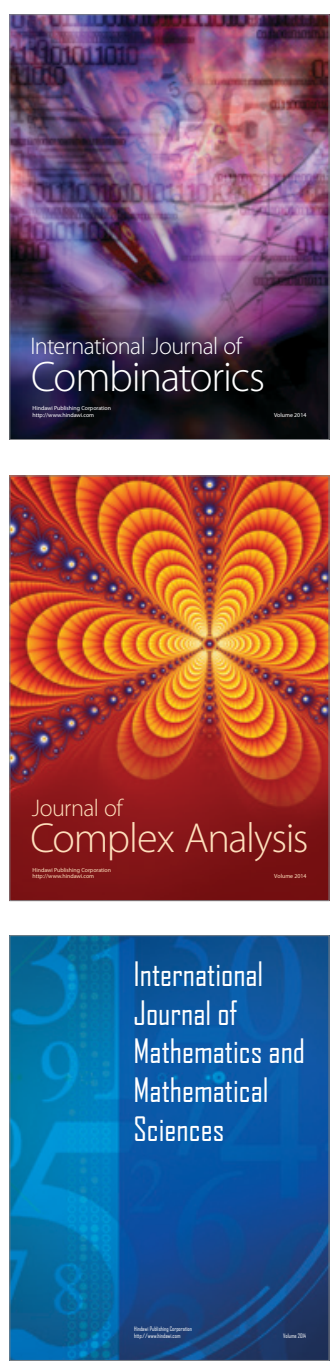
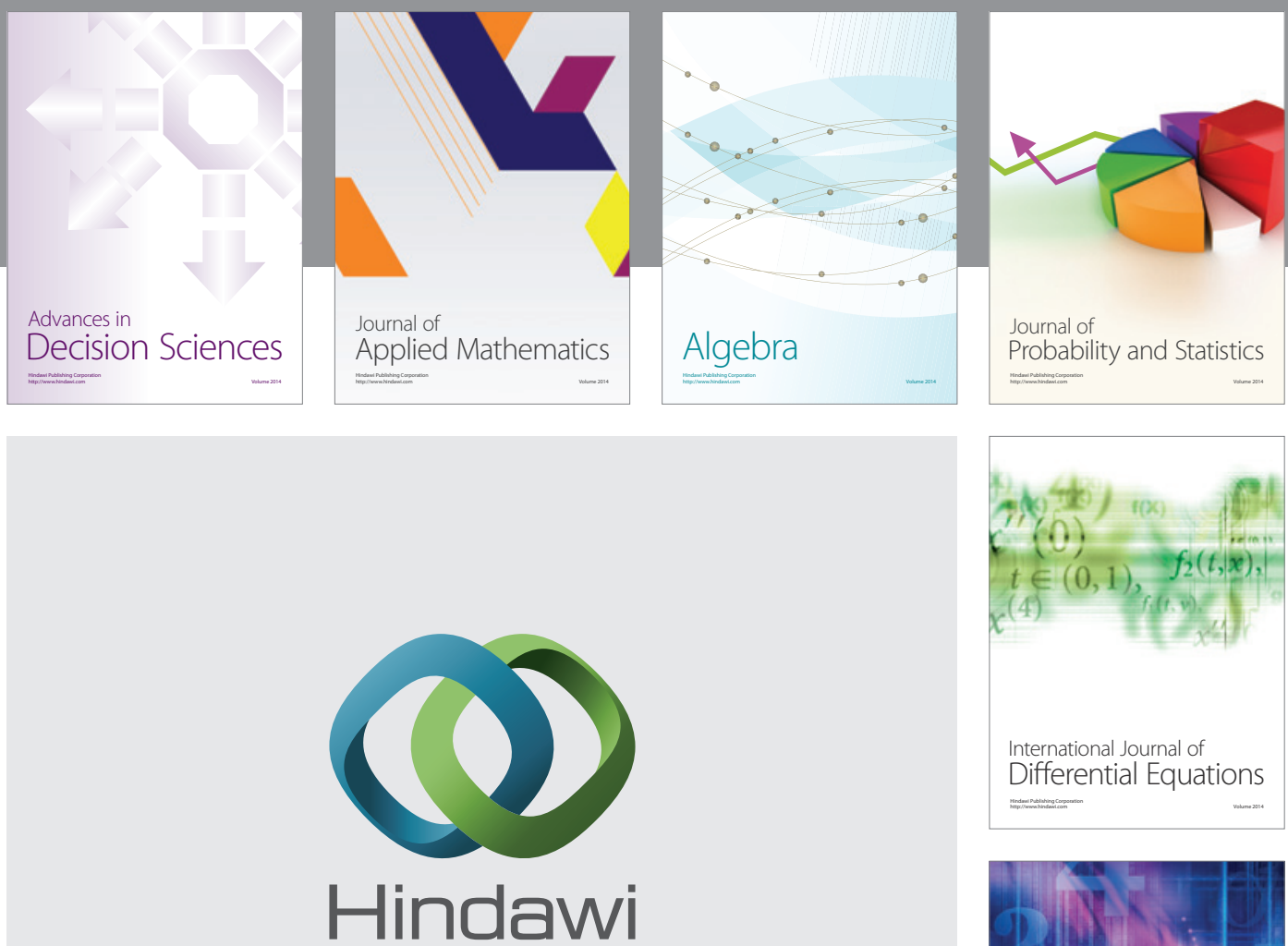

Submit your manuscripts at http://www.hindawi.com
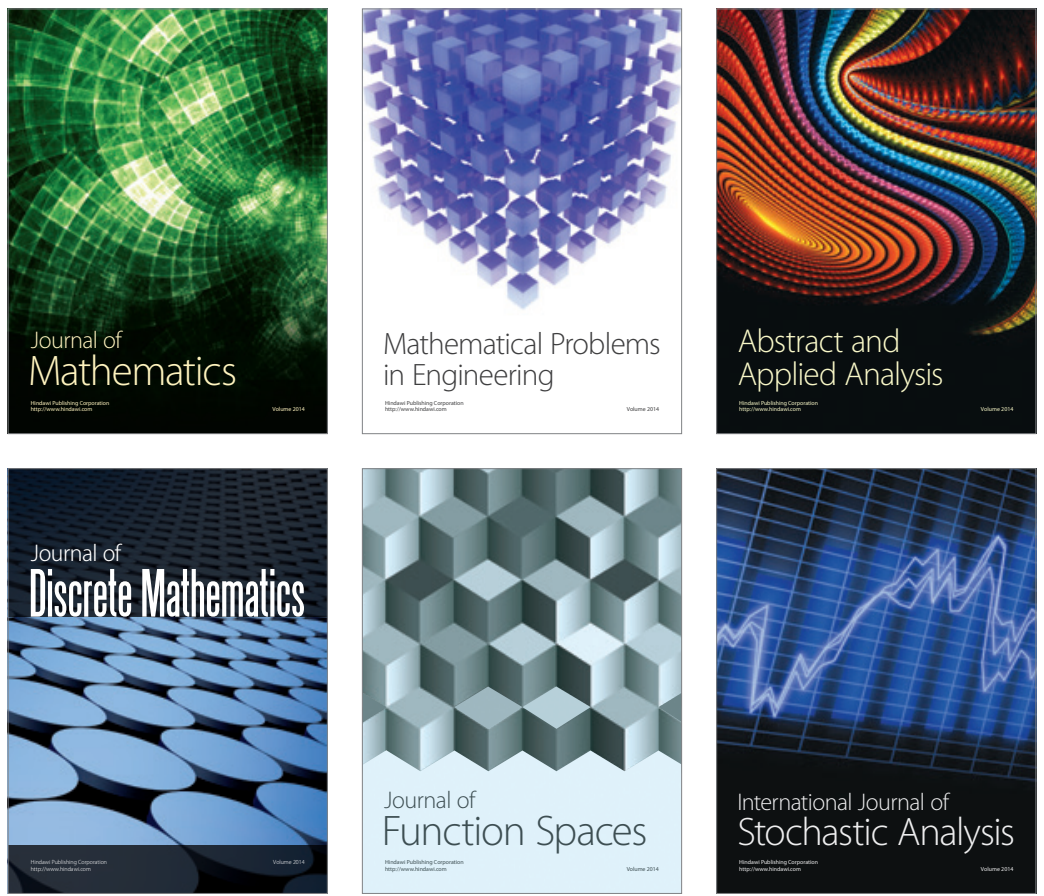

Journal of

Function Spaces

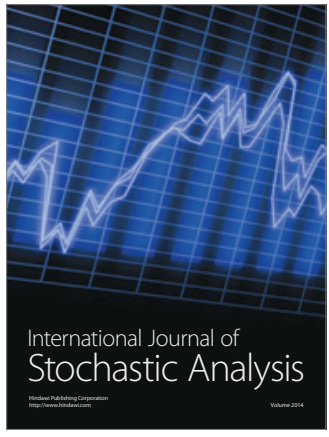

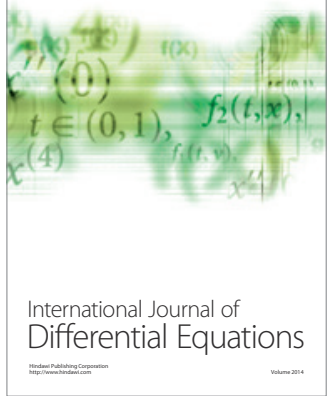
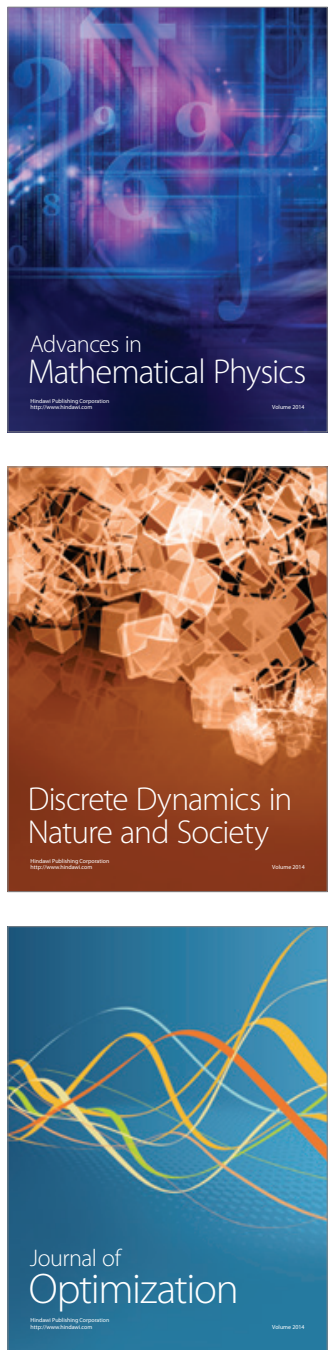